\title{
A FRACTAL MULTI-DIMENSIONAL ULTRASOUND SCATTERER DISTRIBUTION MODEL
}

\author{
Catherine Laporte, James J. Clark and Tal Arbel \\ Centre for Intelligent Machines, McGill University \\ 3480 University Street, Montreal, Qc, H3A 2A7 CANADA
}

\begin{abstract}
This paper presents a multi-dimensional point scatterer distribution model for the context of ultrasound image simulation. The model has a simple parameterisation, has low computational requirements and is flexible enough to model spatial organisation of scatterers ranging from highly clustered to nearly regular. The model extends an existing $1 \mathrm{D}$ model by mapping 1D scatterer positions to a Hilbert space-filling curve. The flexibility of the heuristic model is illustrated through experiments where common statistical models of ultrasonic speckle are fitted to simulated data. The results agree with theoretical predictions.

Index terms: acoustic imaging, simulation, fractals, statistics, speckle
\end{abstract}

\section{BACKGROUND}

An ultrasound (US) image simulator is a useful validation platform for image processing applications where ground truth pertaining to image content or acquisition conditions is needed but not available from US data without additional equipment. It also serves as a test platform during application software development when data acquisition is impossible or awkward. Typical simulators take a list of point scatterers with their strength and position as input along with US transducer specifications and simulate the resulting backscattered signal.

The density and spatial organisation of scatterers are two of many factors which determine the appearance and statistics of US speckle. In order to validate image processing applications or study image properties across different tissue types, it is useful to be able to generate random lists of point scatterers with varying density and spatial organisation, ranging from highly clustered to random to nearly regular. There is currently a lack of models displaying such flexibility along with computational efficiency and simple parameterisation.

The Neyman-Scott point process model has been used for studying the effect of blood cell aggregation on ultrasonic signals [1]. The model creates random cluster centers and spawns daughter points surrounding them. It is not appropriate for generating quasi-periodic patterns. Such patterns can

Catherine Laporte is funded by a doctoral scholarship from the Natural Science and Engineering Research Council of Canada. be generated with varying regularity by perturbing a regular point lattice [2], but this approach cannot generate clustered point patterns. An alternative is a Gibbs-Markov area interaction process which imposes pairwise repulsive and attractive constraints between points. This model was used to study US backscattering from aggregates of non-overlapping blood cells [3]. This type of model is computationally demanding and fails to produce a broad enough variety of clustered patterns when no repulsive constraints are used to maintain a minimal distance between points [4]. In short, previous attempts to parameterise variation in the spatial organisation of scatterers have been geared towards specific applications and have not modeled the full continuum of spatial organisations ranging from clustered to regular. One notable exception is the 1D marked regularity model of Cramblitt and Parker [5]. This model is a generalisation of the Poisson point process with an additional parameter which tunes the variance of inter-scatterer spacing. Unfortunately, the model is one dimensional, and not suitable for 2D or 3D simulations.

This paper proposes a heuristic extension to Cramblitt and Parker's scatterer distribution model for the multi-dimensional case. The proposed approach is to map the results from the original 1D model to a Hilbert space-filling curve. It is shown that this mapping preserves some of the statistical properties of the 1D model. The statistics of resulting simulated images are analysed, showing that the multi-dimensional extension can produce a broad variety of image patterns corresponding to commonly used parametric models of speckle statistics, in agreement with theory.

The rest of this paper is structured as follows. Cramblitt and Parker's 1D model is described in section 2. The proposed multi-dimensional extension is described in section 3 . The validation methods used for experiments are given in section 4 , with the results presented in section 5 .

\section{THE 1D MODEL}

The 1D marked regularity model of Cramblitt and Parker [5] generates a random scatterer function of the form

$$
s(x)=\sum_{i} a_{i} \delta\left(x-X_{i}\right)
$$


where $x$ denotes position on the real line and $a_{i}$ and $X_{i}$ denote the strength and position of scatterer $i$. In this model, the distance $d_{i}=\left|X_{i}-X_{i+1}\right|$ is gamma distributed with shape and scale parameters $\alpha$ and $\beta$ :

$$
f(d)=\frac{d^{\alpha-1} \exp (-d / \beta)}{\Gamma(\alpha) \beta^{\alpha}} \quad \alpha, \beta, d>0 .
$$

This distribution can be usefully reparameterised in terms of the mean and variance in scatterer spacing, $\bar{d}=\alpha \beta$ and $\sigma_{d}^{2}=\alpha \beta^{2}=\bar{d}^{2} / \alpha$. This allows the model to be tuned in terms of scatterer density $(1 / \bar{d})$ and regularity (controlled by $\alpha$ ). With $\alpha=1$, the model reduces to a Poisson point process with exponentially distributed inter-scatterer spacing, and scatterers are placed at random. For $\alpha<1$ (high variance), scatterers tend to group in clusters, whereas for $\alpha>1$ (low variance), scatterers become more evenly spaced.

Incidentally, an identical renewal point process model was independently studied in the field of economic statistics [6]. In this context, it was shown that statistics on the lengths of inter-event (inter-scatterer) intervals have a simple translation in terms of asymptotic statistics on event counts within intervals of fixed length. Specifically, as the length of the fixed interval tends towards infinity,

$$
\frac{E\{N\}}{V A R\{N\}} \rightarrow \alpha,
$$

where $N$ is the scatterer count and $E\{$.$\} and V A R\{$.$\} are the$ expectation and variance operators, respectively.

\section{EXTENSION TO MULTIPLE DIMENSIONS}

While the $\alpha=1$ (Poisson) case is easily generalised to multiple dimensions, there is no obvious extension of the model for other $\alpha$. A simple heuristic to generate a 2D scatterer function would be to rearrange short segments of a 1D scatterer function on a 2D grid in a raster scan fashion. Such a mapping is not desirable because it only preserves the spatial organisation of the points along one direction. The raster scan mapping is but one of many possible mappings of a line segment onto a finite multi-dimensional space known as discrete space-filling curves, which have the property of traversing every single point of a discrete $m$-dimensional grid of a given precision. Better results can be obtained by choosing a different type of space-filling curve with better preservation of locality, such that distances measured along the original line correlate well with distances measured in the multi-dimensional space. A good choice is the Hilbert curve, a fractal curve shown to nearly achieve theoretical bounds in terms of preservation of locality [7]. The Hilbert curve of precision $k$ traverses $\left(2^{k}\right)^{m}$ points on an $m$-dimensional grid. Discrete approximations of the 2D Hilbert curve for different levels of precision are shown in figure 1 .

The algorithm introduced in [9] can be used to determine the Hilbert curve mapping of a point in 1D space to $m$-D

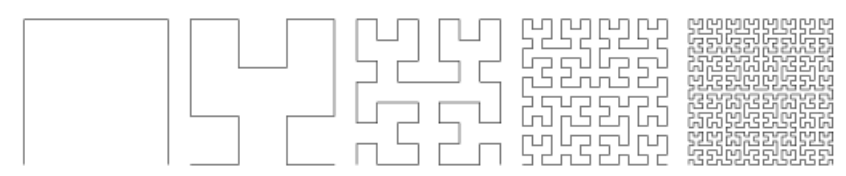

Fig. 1. Discrete approximations of the 2D Hilbert curve [8]. The level of precision of the curves increases from left to right from 1 to 5 .

space. This algorithm takes as input a fixed point $m k$ bit representation of the point's position along the $1 \mathrm{D}$ line segment, recursively removes $m$ bits from the left of this input and uses them as an index to one of $2^{m}$ equally sized sub-cells within the current cell of the $m$-dimensional space. The precision of the resulting $m$-dimensional coordinates is equal to $k$. In 2D or $3 \mathrm{D}$, the process is equivalent to decoding the indexing of cells in a quadtree or octree, respectively.

The Hilbert curve mapping is applied to each point scatterer in the 1D function sampled from Cramblitt and Parker's model, yielding a new set of scatterers in 2D or 3D whose spatial arrangement exhibits similar characteristics to the 1D version. This was empirically verified by generating scattering functions with different parameters $1 / \bar{d}$ and $\alpha$ over large spaces in $1 \mathrm{D}, 2 \mathrm{D}$ and $3 \mathrm{D}$, breaking these spaces into equal numbers of equally sized non-overlapping bins and calculating the ratio of mean to variance in scatterer count over these bins. As predicted by $\mathrm{Eq}$ (3), this ratio is close to $\alpha$ for large bin sizes in the $1 \mathrm{D}$ case. In $2 \mathrm{D}$ and $3 \mathrm{D}$, it was found that the agreement with theory is also good provided that the statistics are measured on full sub-cells of the Hilbert curve (which themselves are Hilbert curves of lower precision). This means that when using the (heuristic) multi-dimensional extension of the model, one should use the data generated for an integer number of full Hilbert curve sub-cells in order to guarantee preservation of the 1D statistics. The agreement between theory and the samples for a 262,144 unit sized space divided into 64 bins is illustrated in figure 2. The agreement was found to be of good quality for all values of $1 / \bar{d}$.

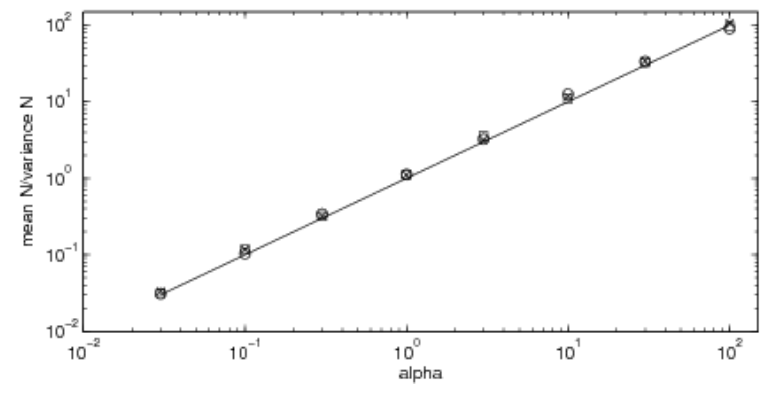

Fig. 2. Ratio of mean to variance in scatterer count versus $\alpha$. Solid line: theory, squares: 1D model samples, circles: 2D model samples, crosses: 3D model samples. 


\section{APPLICATION TO US IMAGE SIMULATION}

The proposed scatterer distribution model was validated on the basis of its usefulness in the context of 2D US image simulation. The model should permit simulation of a broad variety of speckle images. The statistics of these images should be reliably predictable from the model parameters (as with the original 1D model) and in agreement with theoretical considerations.

\subsection{US speckle amplitude statistics}

Theory proposes several parametric models for the statistical distribution of the US echo amplitude $A$. The three main models studied in [5] were retained for this study. The first is the Rayleigh distribution model:

$$
f(A)=\frac{A}{\sigma^{2}} \exp \left(-A^{2} / 2 \sigma^{2}\right)
$$

where $\sigma^{2}$ reflects the variance in scatterer strength. The model assumes a large number of scatterers distributed uniformly in space ( $\alpha$ close to 1 ), resulting in random interference between backscattered waves.

The second model is the $\mathrm{K}$ distribution model:

$$
f(A)=\frac{2 b}{\Gamma(\mu)}\left(\frac{b A}{2}\right)^{\mu} K_{\mu-1}(b A) \quad \mu>0,
$$

where $b=2\left(\frac{\mu}{2 \sigma^{2}}\right)^{1 / 2}$ and $K_{\mu-1}($.$) is the modified Bessel$ function of the second kind and order $\mu-1$. The model is an accurate statistical description of US speckle for low scatterer density or high scatterer clustering $(\alpha<1)$, reflected in the additional parameter $\mu$. The model reduces to a Rayleigh distribution with parameter $\sigma$ for $\mu \rightarrow \infty$.

The third model is the Rician distribution model:

$$
f(A)=\frac{A}{\sigma^{2}} \exp \left(-\left(A^{2}+s^{2}\right) / 2 \sigma^{2}\right) I_{0}\left(\frac{A s}{\sigma^{2}}\right) \quad s \geq 0,
$$

where $I_{0}($.$) is the modified Bessel function of the first kind$ of order 0 , and the parameter $s$ describes the amount of coherent scattering associated with highly organised scatterer structures causing specular reflection and other forms of constructive interference. These may occur for $\alpha>1$. The model reduces to the Rayleigh distribution model for $s=0$.

\subsection{Simulation and model selection}

For quantitative validation, US images were simulated by convolving $1 \mathrm{D}$ and 2D scattering functions with a gated cosine pulse, yielding a radio-frequency (RF) signal. The square pulse envelope was chosen over a Gaussian envelope because the former allows for better defined scatterer/resolution cell count. The resolution cell size $T$ (the same in $1 \mathrm{D}$ and in 2D) was chosen such that the scatterer density, in terms of scatterers per resolution cell, $T / \bar{d}$, varied from 5 to 50 , with $\bar{d}$ set to either 0.5 or 1.0 cycle of the cosine pulse (in $1 \mathrm{D}$, these

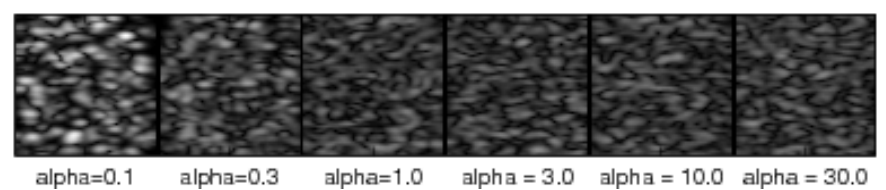

Fig. 3. 2D simulated images obtained with high scatterer density and increasing values of $\alpha$.

correspond to destructive and constructive interference cases, respectively). The regularity parameter $\alpha$ was increased from 0.1 to 30 . The scatterer strengths were independently sampled from a lognormal distribution with mean 1 and variance 0.1 , as suggested in [5]. The amplitude signal was obtained through envelope detection of the RF signal via the Hilbert transform. Ten simulated images of size $1024 T$ were generated for each combination of $T / \bar{d}, \alpha$ and $\bar{d}$.

For each of the three speckle models described earlier, a maximum likelihood fit to the 1024 data samples at the centers of sequential image patches of size $T$ was calculated. A closed form expression for the maximum likelihood value of $\sigma$ exists for the Rayleigh model. The Fletcher-Reeves conjugate gradient algorithm was used to optimise parameters of the Rician distribution model. For the K distribution model, whose log-likelihood function has complicated derivatives, the derivative free Nelder-Mead simplex algorithm was used.

The "best" model was chosen to minimise Schwarz's Bayes Information Criterion (BIC) [10]:

$$
B I C=-2 L+p \log n,
$$

where $L$ is the logarithm of the likelihood function at its maximum, $p$ is the number of parameters in the model analysed and $n$ is the number of data samples. The BIC was chosen over a simple goodness of fit criterion because it measures the complexity of the model in addition to its goodness of fit, choosing simpler models over more complex ones for similar goodness of fit levels. The BIC is particularly appropriate for this study, where the compared models are all generalisations of the simple Rayleigh model.

\section{RESULTS}

For illustration, examples of simulated ultrasound images obtained with high scatterer density and varying $\alpha$ are shown in figure 3. A Gaussian pulse envelope was used for realism, and the images were logarithmically compressed for better visualisation. As $\alpha$ increases and the scatterers are more uniformly placed, it can be seen that the images become smoother.

Figures 4 and 5 show how often each speckle model was chosen out of then ten trials for each simulation case. For small scatterer densities and high clustering $(\alpha<1)$, the $\mathrm{K}$ distribution model is preferred, in agreement with theory, both in 1D and 2D. For random scatterer placement $(\alpha=1)$, small 


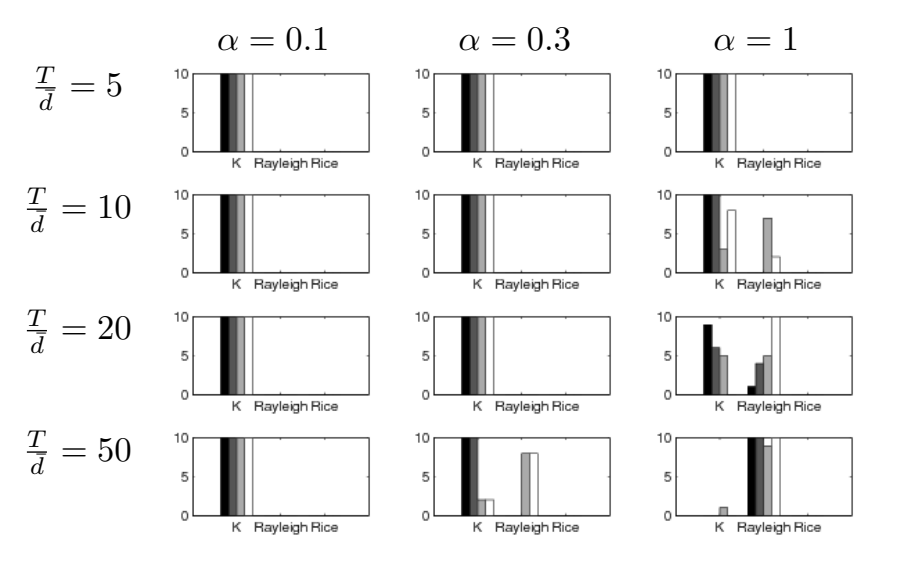

Fig. 4. Number of times each speckle model was chosen out of 10 trials for simulation cases with $\alpha \leq 1$. Black: 1D model, $\bar{d}=1.0$ pulse cycle; dark grey: $1 \mathrm{D}$ model, $\bar{d}=0.5$ pulse cycle; light grey: $2 \mathrm{D}$ model, $\bar{d}=1.0$ pulse cycle; white: $2 \mathrm{D}$ model, $\bar{d}=0.5$ pulse cycle.

scatterer densities yield $\mathrm{K}$ distributed amplitudes, which become Rayleigh distributed as the scatterer density increases. This transition seems to occur for smaller densities in the 2D case than in the $1 \mathrm{D}$ case.

The statistics of the simulated images when regular structure is present $(\alpha>1)$ depend on $\bar{d}$. In $1 \mathrm{D}$, constructive interference occurs for large values of $\alpha$, irrespective of scatterer density, when $\bar{d}=1$ pulse cycle, yielding Rician amplitude statistics. Destructive interference occurs for $\bar{d}=0.5$ pulse cycle, yielding K (for small densities), or Rayleigh (for large densities) distributed statistics. In $2 \mathrm{D}$, the average scatterer spacing $\bar{d}$ is mapped onto a non-linear segment of the Hilbert curve, making it difficult to generate scatterers at exact multiples of half-wavelengths in the direction of wave propagation. However, Rician amplitude statistics can still be obtained with the 2D model for large values of $\alpha$, high scatterer densities and small inter-scatterer spacing. This is illustrated by the 2D case with $\bar{d}=0.5$ pulse cycle. This case effectively simulates a compact, solid structure yielding specular reflection.

While the $1 \mathrm{D}$ and $2 \mathrm{D}$ results may differ for some parameter settings, both make theoretical sense in their context. Moreover, the type of speckle obtained by simulation can be reliably predicted from the parameters of the $2 \mathrm{D}$ model, as it is with the $1 \mathrm{D}$ model.

\section{CONCLUSIONS}

This paper presented a new multi-dimensional scatterer distribution model which can be used in the context of US image simulation. The model builds on a flexible 1D model whose desirable characteristics it preserves well. Simulation results show that textures corresponding to commonly used parametric models of speckle statistics can be obtained from this model and well predicted from the model parameters.

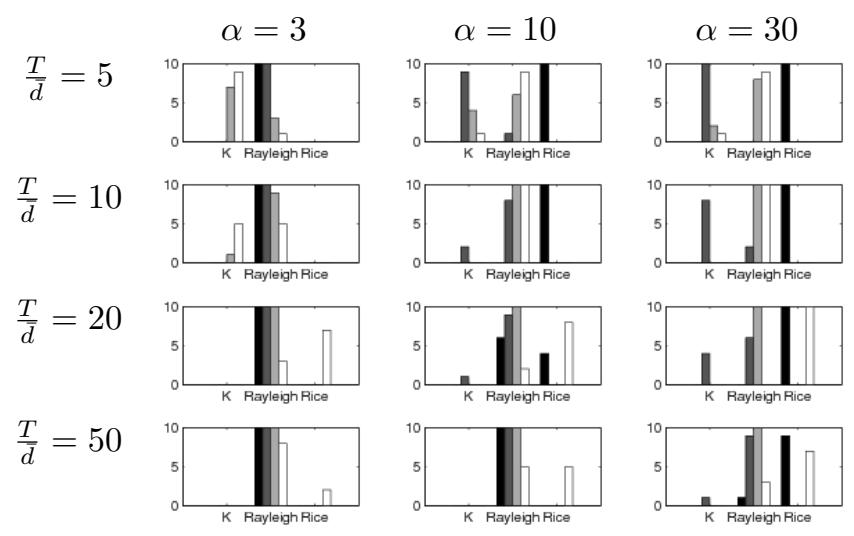

Fig. 5. Number of time each speckle model was chosen out of 10 trials for simulation cases with $\alpha>1$.

\section{REFERENCES}

[1] D. Savéry and G. Cloutier, "A point process approach to assess the frequency dependence of ultrasound backscattering by aggregating red blood cells," $J$. Acoust. Soc. Am., vol. 110, no. 6, pp. 3252-3262, 2001.

[2] V. M. Narayanan, R. C. Molthen, et al., "Studies on ultrasonic scattering from quasi-periodic structures," IEEE T. UFFC, vol. 44, no. 1, pp. 114-124, 1997.

[3] D. Savéry and G. Cloutier, "Effect of red cell clustering and anisotropy on ultrasound blood backscatter: a Monte Carlo study," IEEE T. UFFC, vol. 52, no. 1, pp. 94-103, 2005.

[4] A. J. Baddeley and M. N. M. van Lieshout, "Areainteraction point processes," Ann. I. Stat. Math., vol. 47, no. 4, pp. 601-619, 1995.

[5] R. M. Cramblitt and K. J. Parker, "Generation of nonRayleigh speckle distributions using marked regularity models," IEEE T. UFFC, vol. 46, no. 4, pp. 867-874, 1999.

[6] R. Winkelmann, "Duration dependence and dispersion in count-data models," J. Bus. Econ. Stat., vol. 13, no. 4, pp. 467-474, 1995.

[7] C. Gotsman and M. Lindenbaum, "On the metric properties of discrete space-filling curves," IEEE T. Image Process., vol. 5, pp. 794-797, 1996.

[8] E. W. Weisstein, "Hilbert curve," From Mathworld - A Wolfram Web Resource http://mathworld.wolfram.com/HilbertCurve.html, 1999.

[9] J. J. Bartholdi, III and P. Goldsman, "Vertex-labeling algorithms for the Hilbert spacefilling curve," Software Pract. Exper, vol. 31, no. 5, pp. 395-408, 2000.

[10] G. Schwarz, "Estimating the dimension of a model," Ann. Stat., vol. 6, no. 2, pp. 461-464, 1978. 